\title{
Enquête
}

Archives de la revue Enquête

3| 1996

Interpréter, Surinterpréter

\section{De la surinterprétation des sources diplomatiques médiévales}

Quelques exemples français des alentours de l'an mil

On overinterpretation of medieval deeds. Some French examples around the year one thousand

\section{Olivier Guyotjeannin}

\section{OpenEdition}

Journals

Édition électronique

URL : http://journals.openedition.org/enquete/443

DOI : 10.4000/enquete.443

ISSN : $1953-809 x$

Éditeur:

Cercom, Éditions Parenthèses

\section{Édition imprimée}

Date de publication : 1 novembre 1996

Pagination : 153-162

\section{Référence électronique}

Olivier Guyotjeannin, «De la surinterprétation des sources diplomatiques médiévales », Enquête [En ligne], 3 | 1996, mis en ligne le 11 juillet 2013, consulté le 22 avril 2019. URL : http:// journals.openedition.org/enquete/443; DOI : 10.4000/enquete.443

Ce document a été généré automatiquement le 22 avril 2019 


\title{
De la surinterprétation des sources diplomatiques médiévales
}

\author{
Quelques exemples français des alentours de l'an mil \\ On overinterpretation of medieval deeds. Some French examples around the year \\ one thousand
}

Olivier Guyotjeannin

1 Assez fournie pour attirer l'historien et assez lacunaire pour laisser une large place à la reconstitution, la documentation d'archives occidentales des $\mathrm{X}^{\mathrm{e}}$ et $\mathrm{XI}^{\mathrm{e}}$ siècles obéit à des codes, à des stratégies de mise par écrit, que la diplomatique commence à peine à prendre en compte avec l'attention qu'elles méritent. Née au XVII siècle, la discipline s'était d'abord donné pour tâche de séparer, parmi les diplômes de souverains, plus largement dans l'ensemble des actes écrits, le vrai du faux, l'acte sincère de la forgerie. Attentive bientôt à reconstituer les modalités de travail au sein des chancelleries, la formation des écrivains professionnels, plus tard soucieuse de dégager des règles d'édition des documents et d'analyse de leur tradition, elle a inévitablement été soumise, dans son programme, aux vues dominantes sur les fins de l'histoire (apologétique, défense du prince puis de la Nation, illustration de l'idée de progrès...), dans ses présupposés, aux périodisations proposées par les historiens.

Nulle époque sans doute ne le montre mieux que les $\mathrm{X}^{\mathrm{e}}$ et $\mathrm{XI}^{\mathrm{e}}$ siècles, tenus pour le moment des grands bouleversements souterrains et cataclysmiques, âge moyen du Moyen Âge. Au paradigme longtemps dominant de l'« anarchie féodale » (genèse tourmentée, dans le bruit et la fureur, d'un ordre nouveau qui se reconstruira par le bas après avoir jeté bas toutes les structures publiques d'encadrement, mettant dans l'œil du cyclone l'«impuissant " Hugues Capet), le diplomatiste a longtemps emprunté la vision d'une période produisant peu d'écrit, et mal. Le diplôme royal $\mathrm{du} \mathrm{XI}$ siècle capétien est-il (parfois) couvert de souscriptions de tiers, c'est symptôme de faiblesse royale, à l'image d'un prince qui doit « demander conseil » à ses grands ou à ses familiers. L'acte privé estil rare dans nos archives, établi avec une incroyable variété de formats, de mises en page, 
d'écritures, de présentation, de vocabulaire, c'est encore preuve d'«anarchie », ou de «maladresse».

3 Au terme d'un processus séculaire caractérisé par la reproduction et la réappropriation de formules (vocabulaire, parties du discours, cadre général de l'acte) héritées de la Basse Antiquité, à l'aube d'une autre période qui sera celle de toutes les créations (juridictions gracieuses et notariat public, acte de souverain et acte pontifical régénérés, acte épiscopal «inventé »...), et de toutes les mises au point qui s'ensuivent (surgissement de l'anthroponymie moderne, apparition du réseau de microtoponymes, vocabulaire renouvelé, apparition de langues techniques - latines ou vernaculaires - fortement imprégnées d'un droit qui ne peut être que savant), les documents produits autour de l'an mil sont révélateurs d'une période de transition. Du coup, ils offrent un vaste champ à un examen renouvelé... et des occasions uniques à la surinterprétation : lexique, tournures, formules, contenu du discours ont tous été appelés au secours de raisonnements circulaires, qui faisaient miraculeusement trouver en fin d'enquête l'illustration des présupposés qui avaient été à l'origine de celle-ci. Et ce d'autant que, selon un préjugé fortement ancré, les sources d'archives, à la différence des témoins littéraires, «ne mentent pas»: malentendu nourri depuis l'époque même de la naissance de la diplomatique, dont Mabillon, il y a plus de trois siècles, faisait un « art de distinguer le vrai du faux » (discrimen veri ac falsi), engageant du coup des disciples moins subtils, plus spécialisés que lui dans cet unique champ documentaire, à croire que la diplomatique, décernant un label d'authenticité à certaines sources, validait aussi, sans autre forme de procès, des témoignages pour l'historien.

4 Les sources diplomatiques des alentours de l'an mil, dans les régions aujourd'hui françaises (en dépit de leur diversité à l'époque) - disons dans l'espace de ce qui est alors le « royaume des Francs de l'Ouest », de la Flandre à la Catalogne -, offrent une spécificité certaine, que l'on a encore trop peu mise en parallèle avec l'historiographie des mêmes régions : or, les thèmes de l'« anarchie féodale », des " terreurs de l'an mil », sont presque uniquement français. Dans l'époque, les historiens de langue allemande sont allés longtemps rechercher la construction d'un bel ordre étatique (la "renaissance» ottonienne) domptant les hordes hongroises et quelque trublion bavarois. On voudrait suggérer que ces différences de lecture ont largement reposé sur des différences de la base documentaire.

5 Différences qui sont, d'abord, de quantité. Hugues Capet, roi de Francie occidentale de 987 à 996, n'a laissé qu'une petite quinzaine d'actes (mis à part ceux sur qui pèsent de lourds soupçons de falsification). Otton III, roi de Germanie et d'Italie (du nord), maître plutôt contesté de Rome et empereur magnifié par une propagande qui s'intensifie, de 983 à 1002, en a laissé environ 420. Autant les premiers varient dans leur formulaire, dans leur présentation, autant les seconds présentent une vaste gamme de traits communs. À Paris ou Senlis, un chapelain, parfois le bénéficiaire lui-même, dresse le diplôme capétien; à Aix, Rome ou Magdebourg, une chapelle-chancellerie industrieuse, vivier d'évêques, dit sans relâche la gloire de l'empereur (ce qui n'empêche d'ailleurs pas certains actes d'être aussi produits, de façon incertaine, par les bénéficiaires, le souverain d'en souscrire tout simplement d'autres, non établis en son auguste nom: mais ceux-là, les diplomatistes allemands les taisent, les réputant « non royaux »).

6 Les actes "privés ", qui enregistrent les plus modestes des transactions de base de la vie en société sont aussi divers et rares dans le territoire français qu'ils sont répétitifs et nombreux dans les innombrables chartriers du nord de l'Italie. Lire dans le petit nombre 
d'actes, incroyablement variés dans leur expression et leur présentation, des régions aujourd'hui françaises les symptômes d'une "baisse de la culture écrite", en même temps que d'une anarchie sociale, en contraste avec l'ordre ottonien, avec la culture écrite italienne, et qui marquerait un "siècle de fer ", a été longtemps un lieu commun des études diplomatiques et historiques.

Ce que faisant, la diplomatique oubliait l'une de ses missions : non seulement distinguer l'acte sincère de l'acte forgé quelques décennies ou quelques siècles plus tard, mais encore dégager sa genèse, le contexte qui l'a vu naître et les modalités concrètes de sa rédaction; et, plus largement, soupeser le « besoin d'écrit » qui l'a suscité et réfléchir à la façon dont il a pu être conservé ou éliminé. Si l'on procède à cet examen, qui en est encore à préciser ses méthodes et à ordonner ses conclusions, on constate sans peine que la documentation "française » enregistre, précisément au cours des $\mathrm{X}^{\mathrm{e}}$ et $\mathrm{XI}^{\mathrm{e}}$ siècles, une mutation fondamentale. Aux vieux formulaires appliqués par des dynasties d'écrivains professionnels, réunis par une commune formation si ce n'est par la filiation, et pour qui la reproduction des vieilles recettes était garante d'authenticité, elle substitue un discours différent, tenu par l'une des parties, le bénéficiaire même de l'acte, dont les archives sont aujourd'hui les seuls réservoirs de sources diplomatiques. Discours nouveau dans ses rythmes et son vocabulaire, dans sa narration et son ordre; discours tout autant codé, pas moins stéréotypé, mais avec d'autres codes et d'autres stéréotypes. On y gagne une documentation plus colorée, où Dominique Barthélémy a récemment dégagé le concept fort opératoire de "révélation documentaire ${ }^{1} »$ : une révélation qui a pu laisser accroire que la société se métamorphosait au rythme des apparitions (d'ailleurs contradictoires) d'un terme ou d'une situation qui ne se rencontraient pas auparavant dans la documentation écrite. Une documentation qui s'ouvre à de nouvelles formes, à tâtons (d'où l'impression de bricolage qui transparaît de nombreuses productions), en se forgeant peu à peu des traditions locales ou régionales.

8 Si l'on ajoute, pour reprendre la comparaison avec la documentation italienne, que l'historien français ne lit plus que les documents qui ont trouvé grâce aux yeux des réformateurs monastiques $\mathrm{du} \mathrm{XI}^{\mathrm{e}}$ siècle et des gestionnaires avisés du siècle suivant, dont les cartulaires ont impitoyablement sélectionné la documentation, alors que les -

chartriers italiens ont largement vécu sur le mode de la continuité et de la préservation de l'original, de tout original, on comprend mieux que les deux zones présentent aujourd'hui des situations si différentes².

Depuis plus d'un siècle, les diplomatistes de l'époque positiviste ont fait justice du mythe des «terreurs de l'an mil », qui reprend un peu de vigueur en notre fin de millénaire plus exactement de ses prétendues attestations dans les actes. Il s'agit d'un véritable cas d'école: aux historiens romantiques qui avaient monté en épingle les préambules de quelques actes dont le rédacteur avançait, pour justifier une donation, la prochaine fin du monde (Adpropinquante mundi termino...), ils ont opposé la permanence d'un fonds de préambules et de considérations, remployé par les rédacteurs d'actes $d u \mathrm{VI}^{\mathrm{e}}$ à la fin du XI ${ }^{\mathrm{e}}$ siècle et, plus largement, la prégnance d'une vue "médiévale » de l'histoire du monde comme celle d'un déclin, d'un vieillissement irrémédiables et rapides ${ }^{3}$. Le rédacteur d'actes mérovingien était déjà persuadé que le monde allait à sa perte (et à son Jugement), à tout le moins y voyait-il, comme ses collègues, une entrée en matière suffisamment conforme à la culture du lecteur pour être incorporée à sa production.

Plus complexe, la légende de l'« anarchie » s'est aussi plus subtilement appuyée sur une identique surinterprétation des sources documentaires. C'est sans doute qu'elle était trop 
bien liée à une certaine représentation des siècles médiévaux dont les acquis ont été, par ailleurs, essentiels à la conception et à l'essor de l'histoire "médiévale». Depuis les premières décennies du XIX siècle (que l'on pense par exemple aux «temps mérovingiens » d'Augustin Thierry), le Moyen Âge a dû gagner son identité en brisant, par la grâce des invasions, mutations et autres révolutions, la tranquille continuité qui le noyait jusque-là dans un long fleuve au cours tranquille: continuité providentielle des trois races de rois, identité nationale et chrétienne... L'avènement de Hugues Capet, l'apparition d'un vocabulaire nouveau dans les actes, un peu de peurs et beaucoup de violences révélées par des sources en fait plus disertes : tout cela coïncidait trop bien pour ne pas inciter à tirer de la documentation l'idée d'un bouleversement sauvage des bases sociales. On a dit combien les $\mathrm{X}^{\mathrm{e}}$ et $\mathrm{XI}^{\mathrm{e}}$ siècles (au juste, surtout le second), avec leur "révélation documentaire ", disent plus de violence, sans en vivre forcément davantage.

11 Prenant le contre-pied de ces vues, des historiens insistent sur la permanence quasi fixiste des structures antiques. Partis d'un examen de sources réputées « fiscales » parce qu'elles persistent à employer des termes de la Basse Antiquité, certains déduisent, de la permanence du lexique, la pérennité des structures d'administration ${ }^{4}$. Partis d'un autre problème et d'autres sources, des historiens, à l'origine historiens du droit, traquent depuis plus d'un siècle les moindres signes d'une « connaissance du droit romain » dans les sources documentaires ${ }^{5}$. Souvent séparés dans leurs centres d'intérêt ou leurs conclusions, ils ont en commun l'insistance mise sur les continuités. De telles études ont le mérite de corriger la vision sauvage de souverains du $\mathrm{X}^{\mathrm{e}}$ ou XI $\mathrm{XI}^{\mathrm{e}}$ siècle, grossiers et incultes chefs de clan, et d'inciter à dépasser l'anachronique opposition entre public et privé, mais elles succombent, dans une destination opposée, aux mêmes erreurs de méthode.

Dans le premier domaine, le moindre terme extrait des documents sert à prouver, par exemple, que les Capétiens du $\mathrm{XI}^{\mathrm{e}}$ siècle disposaient d'une organisation fiscale et d'une comptabilité dont on se demande en quoi les siècles postérieurs auraient pu l'améliorer. Or, si un souverain du XI ${ }^{e}$ siècle donne à des moines les revenus d'un marché, la moitié du péage d'un pont, est-ce forcément qu'il en lit et contrôle chaque année la comptabilité ? Quand le roi Philippe $\mathrm{I}^{\mathrm{er}}$ en 1071 donne à l'évêque de Laon quicquid de regio computatur apud villam que Vallis vocatur, est-ce que nécessairement il « existait des comptes annuels par villa» et que "les rois pouvaient, lors de leurs innombrables et nécessaires déplacements, se faire présenter toutes les pièces comptables qu'ils désiraient vérifier ou faire vérifier ${ }^{6} »$ ? N'est-ce pas plutôt que l'on tiendrait l'une des premières attestations, sous la plume du clerc qui rédige l'acte, d'une tournure latine nouvelle, passée à la langue française (plutôt même que d'une tournure vulgaire latinisée) : « tout ce que l'on compte de royal à Vaux ». Et donc, en poussant l'analyse, que l'on aurait là l'un des nombreux témoignages de ce mélange de solennité et d'imprécision, de majesté et de flou, d'absolu (dans le pouvoir affirmé) et d'incertain (dans la domination sur la terre), qu'un diplomatiste allemand, Peter Rück, a par ailleurs si magistralement dégagé des actes ottoniens? Questions qui font toucher du doigt le processus d'élaboration d'un langage, le langage des actes, et qui sont au moins aussi captivantes que de demander si Hugues Capet est successeur de Dioclétien.

Dans le second domaine, celui de l'histoire du droit, on sait mieux aujourd'hui que, s'il y a eu une «redécouverte » du droit romain au $\mathrm{XII}^{\mathrm{e}}$ siècle, elle a été sélective, orientée et oublieuse de "redécouvertes" partielles et antérieures; que certains textes du droit romain tardo-antique avaient été lus, copiés, glosés bien avant. Mais ici encore la traque 
des moindres adages, passablement déformés, employés parfois à contresens, aboutit à un appauvrissement de la véritable question : quel usage, quelle source précise un rédacteur d'acte a-t-il en vue ? La question de la lèse-majesté en donne une illustration. Le XII ${ }^{\mathrm{e}}$ siècle n'a pas exhumé le concept de siècles lointains; il l'a systématisé pour en tirer des conséquences plus rigoureuses. Du coup, voir le terme fleurir dans quelques actes ou lettres de la fin du $\mathrm{X}^{\mathrm{e}}$ siècle capétien prête moins à conséquence pour l'historien, et davantage pour le diplomatiste et l'historien de la diffusion des textes, qui reconstitue des filiations, des lectures, en même temps qu'il y voit l'une des multiples manifestations de l'imprégnation des rédacteurs par un savoir scolaire, attentif aux termes rares comme aux fleurs de la rhétorique ${ }^{7}$. Un comte sera qualifié de consul; le roi de France Robert le Pieux sera, ailleurs, dit augustus. Y voir la prégnance du monde antique ou l'émergence d'une idée impériale chez le deuxième Capétien ne tient pas.

Un acte de Hugues Capet permet de saisir, plus profondément que d'autres, tout le jeu de la surinterpération des sources diplomatiques. En 993, le roi délivre un précepte à l'abbaye de Fleury (Saint-Benoit-sur-Loire) que, dès sa première année de règne, il avait ornée d'un privilège d'immunité. Autant l'acte de 987 était d'une rédaction stéréotypée (il reprenait d'ailleurs très largement la teneur d'actes royaux carolingiens), autant celui de 993 provoque le lecteur ${ }^{8}$ :

«Au nom de la sainte et indivise Trinité. Hugues, par la grâce de Dieu roi des Francs. L'usage et la coutume des rois nos prédécesseurs ont toujours été d'élever les églises de Dieu, de répondre avec clémence aux justes demandes des serviteurs de Dieu et de les décharger avec bienveillance des oppressions qu'ils subissaient, pour s'attirer la faveur de Dieu, par amour de qui ils agissaient ainsi. Pour cette cause, j'ai entendu les plaintes du vénérable Abbon, abbé du monastère de sainte Marie, saint Pierre et saint Benoît de Fleury, et des moines qui vivent sous ses ordres, qui sont venus en notre présence, au sujet des mauvaises coutumes et rapines incessantes qu'Arnoul du château d'Yèvre prenait, sous couvert de l'avouerie et de la voirie, dans leur pôté [domaine] appelée Yèvre, ce qu'auparavant personne n'avait jamais fait. J'ai envoyé en ce lieu mon fils le roi Robert pour qu'il la remette sous notre défense et protection, afin qu'aucun de ses hommes, non libre ou fibre, n'ose rien y prendre; ce qu'il a fait avec diligence. Mais entre-temps le comte Eudes s'est levé contre moi et, parmi tous les alliés et fidèles que nous avons réunis par semonce autour de nous, nous avons aussi fait venir à notre aide Arnoul, évêque d'Orléans, qui a, pour cette raison, demandé que nous restituions les coutumes au susdit Arnoul, son neveu, comme il les avait tenues auparavant, quoique violemment. Ne voulant, pour son service, l'offenser, j'ai rappelé le susdit abbé et lui ai demandé de verser, [des revenus] de ladite pôté, trente muids de vin, aux vendanges, audit Arnoul, tant que vivrait son oncle l'évêque, pour notre sauvement, à cette condition qu'aucun des hommes [d'Arnoul], non libre ou libre, n'y exigerait davantage ; et qu'après la mort de [l'évêque], ni ledit Arnoul ni aucun de ses successeurs n'aurait l'audace de réquisitionner, dans ladite pôté, ni cela ni quelque autre chose, ni la présomption d'y entrer et d'en enlever désormais quoi que ce soit. Ce que pour renforcer et notifier, $\mathrm{j}$ 'ai fait faire pour ledit monastère, pour cette cause, le présent précepte de notre immunité, je l'ai ci-dessous renforcé de ma propre main et je l'ai fait renforcer par mon fils, le roi Robert, et je l'ai marqué de l'empreinte de mon sceau, afin qu'à l'avenir personne jamais, évêque, abbé, duc, comte, vicomte, voyer, tonloyer ou tout autre collecteur public ne puisse $\mathrm{y}$ entrer pour y collecter quoi que ce soit, de notre temps ou aux temps à venir, et n'ait la présomption d'y collecter quoi que ce soit, mais que l'abbé et la congrégation du susdit monastère, défendus par notre immunité, puissent en toute quiétude posséder ladite possession; et que ce bien, comme tous les autres biens du susdit monastère, protégés et défendus par les préceptes des rois des Francs nos prédécesseurs, reste lui aussi irrévocablement protégé par la présente autorité de 
notre précepte, pour le bien de mon âme et de l'âme de mon fils et pour la

perpétuelle stabilité de notre pouvoir souverain. »

Le rappel normalement insistant du pouvoir royal, le prologue passe-partout, la fin de l'acte coulé dans un formulaire usuel, forment contraste avec le récit précis mais dépouillé, parfois virulent, presque cru, qui occupe la plus grande partie du document. Un méchant châtelain qui « envahit » les possessions des moines désarmés, et voilà pour la violence déchaînée. Un évêque armé qui négocie son service, un roi qui quémande les fidélités, qui revient même sur la parole d'abord donnée à un abbé, et voilà l'impuissance du roi (de l'État !). En 1854, Eugène de Certain entend parler, dans l'acte, une royauté qui " fait elle-même l'aveu de sa faiblesse »; moins d'un demi-siècle plus tard, Ferdinand Lot écrit que cette " petite anecdote» (sic) montre un « roi mal obéi ». L'interprétation en reste naturellement là si elle prend l'acte pour ce que l'histoire positiviste en fait: un témoin qui dit (pauvrement) la vérité.

perspective peut être renversée. Par chance en effet, l'original de l'acte est conservé. La comparaison de ses caractères externes, comme le dit le jargon diplomatique (format, écriture, signes de validation...), comme de certaines formules rédactionnelles, avec les autres diplômes de Hugues Capet permet de penser que le document n'a pas été rédigé par un chapelain du roi, mais par un moine de Fleury (aujourd'hui Saint-Benoît-surLoire). Du coup, tout s'éclaire. Recourant largement à des termes et à des concepts bien attestés dans la production normative du IXe siècle (la « violence » et l'« invasion » sont moins de sauvages coups de main que des détournements de revenus, dans le cadre de dominations incertaines que l'on s'efforce de faire sanctionner par un pouvoir supérieur, ou par la coutume), le rédacteur monastique cherche à consacrer par l'écrit (un écrit presque préparé à la sauvette) le résultat d'une très instable transaction, où le roi a dû tempérer et temporiser, où l'abbé a dû composer.

Moine qui juge les hommes du siècle (évêques compris) comme ceux qui n'ont pu ou su se détacher du monde, le rédacteur a voulu, par un acte qui est à la fois récit et instrument de droit, consacrer les strictes limitations temporelles et matérielles mises au demi-échec de son abbé. Si l'on confronte le document aux autres sources (toutes monastiques) relatives à l'abbaye, on perçoit aussi en négatif tout ce que le document ne dit pas: engagé dans une lutte implacable contre l'ordinaire qui exige la soumission de l'abbaye à l'évêque, Fleury est aussi largement soumis au pouvoir royal, qui imposera quelques années plus tard, non sans grave tension, un nouvel abbé. Toute la réussite du rédacteur est dans la théâtralité de sa mise en scène. Car il lui faut taire les motifs bien précis et assurément pesants que le roi avait de faire avaler à l'abbé une amère potion; il lui faut donc détourner les regards, insister sur les motifs du revirement du roi, dresser le tableau circonstancié des tergiversations du souverain et du chantage à la fidélité qu'exerce sur lui l'évêque d'Orléans. Roi faible et fidèle intrigant, prompt à caser un neveu? Certes oui, mais sans doute pas plus qu'au IX ${ }^{\mathrm{e}}$ ou qu'au XVI ${ }^{\mathrm{e}}$ siècle!

Ce rapide examen permet de constater qu'ici aussi la surinterprétation n'est jamais que sous-interprétation. Mais en dépit de leur position usuelle sur l'échiquier des sources historiques, les sources diplomatiques gagnent, non seulement à risquer le jeu de la « surcompréhension ", si l'on peut reprendre l'expression à Jonathan Culler', mais encore à être traitées comme des sources "littéraires", des récits dont le responsable, avec plus ou moins d'art et de contrainte, doit trouver un compromis entre formulaire et situation précise, entre des conventions imposées par la société et une marge de liberté, grosse à la fois de promesses et de dangers : promesses et dangers d'un écrit qui fera d'autant plus 
foi auprès des contemporains qu'il aura su capter leur attention, emporter leur conviction par l'adaptation habile des codes généraux à la description ponctuelle, par un incessant travail d'ajustement entre tradition et innovation.

L'école diplomatique de langue allemande a apporté de magnifiques études en replaçant l'acte de souverain du haut Moyen Âge dans le cadre de sa réception : rhétorique de l'acte lu, si ce n'est psalmodié, en public; «rhétorique visuelle » de l'acte exhibé ${ }^{10}$. Les études commencent à peine à prendre en compte l'élaboration du " récit $^{11}{ }$. Ce que vient, par exemple de faire Michel Zimmermann dans une admirable analyse des "formules de pertinence ", où le rédacteur énumère de façon aussi vaste et exhaustive que possible tous les types de biens, arbres et moulins, champs et cours d'eau, espaces incultes et espaces « à découvrir ", dont peut se composer le bien-fonds qui fait l'objet de l'acte écrit ${ }^{12}$. Loin d'être témoins de richesse agraire (surinterprétation encore courante), loin d'être fatras routinier (degré zéro de la surinterprétation chez les diplomatistes positivistes), ces formules, mises en série, interprétées comme un document d'histoire culturelle, peuvent ainsi révéler, pour faire bref, l'œil et l'art du notaire ; l'évolution du premier, l'étendue du second. À poursuivre le jeu, on s'apercevra que les sources diplomatiques ont bien plus à livrer que le morne catalogue de noms et de dates, de lieux et d'actions que l'on a coutume d'y puiser.

\section{NOTES}

1. D. Barthélémy, La société dans le comté de Vendôme, de l'an mil au XIVe siècle, Paris, Fayard, 1993, p. 9-116.

2. Sur ce problème, pour faire bref, on se permet de renvoyer à différents dossiers étudiés dans : O. Guyotjeannin et E. Poulle, eds, Autour de Gerbert d'Aurillac, le pape de l'an mil : album de documents commentés, Paris, École nationale des chartes, 1996; O. Guyotjeannin, L. Morelle, M. Parisse, eds, Les pratiques de l'écrit documentaire au XIe siècle, numéro spécial de la Bibliothèque de l'École des chartes, CLV, 1997, fasc. 1. Les quelques réflexions qui suivent leur doivent beaucoup.

3. La critique est faite de façon remarquable par A. Giry, Manuel de diplomatique, Paris, 1894, p. 543-544, avec la pointe qu'il faut d'anticléricalisme: "Il n'y a là rien autre chose que l'expression banale de la doctrine catholique sur la proximité de la fin du monde, très propre à être invoquée par les moines pour déterminer les laïques à se dépouiller de leurs biens. »

4. J. Durliat, Les finances publiques de Dioclétien aux Carolingiens (284-889), Sigmaringen, Thorbecke, 1990, tire par exemple argument de la permanence du terme descriptio pour en inférer la permanence de la cadastration fiscale. À confronter, sur un cas précis, avec l'étude fine de l'élaboration progressive d'un type documentaire: J.-P. Devroey, «Les premiers polyptyques rémois, VIII $-\mathrm{IX}^{\mathrm{e}}$ siècles ", in A. Verhulst, ed., Le grand domaine aux époques mérovingienne et carolingienne, Gand, Centre belge d'histoire rurale, 1985, p. 78-97.

5. D. P. Block, «Les formules de droit romain dans les actes privés du haut Moyen Âge ", in Miscellanea mediaevalia in memoriam Jan Frederik Niermeyer, Groningen, 1967, p.17-28 (avec bibliographie).

6. E. Magnou-Nortier, "Pouvoir, finances et politique des premiers Capétiens », in Id. ed., Pouvoirs et libertés au temps des premiers Capétiens, Maulévrier, 1992, p. 150. L'acte est édité par 
M. Prou, Recueil des actes de Philippe ${ }^{\text {er }}$, roi de France, Paris, Académie des inscriptions et belleslettres, $1908, \mathrm{n}^{\circ} 61$.

7. J.-P. Poly, «Le sac de cuir : la crise de l'an mil et la première renaissance du droit romain ", in J. Krynen et A. Rigaudière, Droits savants et pratiques françaises du pouvoir (XI ${ }^{e}-X V^{e}$ siècles), Bordeaux, Presses de l'université de Bordeaux, 1992, p. 39-68. On pourrait dire la même chose du concept de res publica: le trouver dans des actes carolingiens n'est pas inintéressant, déduire d'une baisse des attestations ultérieures que l'idée d'État se perd est nettement plus audacieux, surtout si l'on a survalorisé l'observation précédente.

8. Dernière édition du texte latin par M. Prou et A. Vidier, Recueil des chartes de l'abbaye de SaintBenoit-sur-Loire, t. I, Paris, 1900-1907, nº LXX, p. 182-185.

9. Ou plus exactement à sa traduction française : J. Culler, « Défense de la surinterprétation », in U. Eco, Interprétation et surinterprétation, Paris, Presses universitaires de France, 1996, p. 106.

10. Ainsi, entre autres exemples, H. Fichtenau, «Bemerkungen zur rezitativischen Prosa des hochmittelalters ", in A. Haidacher et H. E. Mayer, eds, Festschrift Karl Pivec, Innsbruck, 1966, p. 21-32 ; P. Rück, «Die Urkunde als Kunstwerk», in A. von Euw et P. Schreiner, eds, Kaiserin Theophanu, Cologne, Schnutgen Museum, 1991, t. II, p. 311-333.

11. H. H. Kortüm, Zur päpstlichen Urkundensprache im frühen Mittelalter: die päpstlichen Privilegien, 896-1046, Sigmaringen, Thorbecke, 1995, renouvelle ainsi profondément la compréhension de la production des actes pontificaux et, partant, des manifestations et des initiatives de la papauté en dévoilant, sur des bases linguistiques, la part prise par les bénéficiaires à la rédaction des actes.

12. M. Zimmermann, «Glose, tautologie ou inventaire? L'énumération descriptive dans la documentation catalane du $\mathrm{X}^{\mathrm{e}}$ au XII ${ }^{\mathrm{e}}$ siècle ", Cahiers de linguistique hispanique médiévale, 14-15, 1989-1990, p. 309-339. La même démarche a été appliquée avec un identique bonheur à une recherche en matière d'histoire du bâti : E. Hubert, Espace urbain et habitat à Rome du $X^{e}$ siècle à la fin du XIII siècle, Rome, École française de Rome, 1990.

\section{RÉSUMÉS}

Le faible nombre des actes écrits dans la France des $\mathrm{X}^{\mathrm{e}}$ et $\mathrm{XI}^{\mathrm{e}}$ siècles, leur diversité, leurs nouveaux modes de présentation ont été longtemps objet de surinterprétations chez les diplomatistes qui y ont appliqué à toute force les paradigmes dominants de l'historiographie. Sous-interprétations, plutôt, qui ont masqué les témoignages les plus captivants : procédures et enjeux de la mise par écrit de litiges, de compositions, de donations, qui gagent au jeu de la « surcompréhension ».

The small number of written documents drawn up in 10th and 11th century France and the diversity of new styles of presentation, were for a long time a subject of overinterpretation with specialists of diplomatics, who applied the dominant paradigms of historiography at all costs. Under-interpretations, which have concealed, to a certain extent, the most fascinating evidence of all: proceedings and stakes in the drawing up of lawsuits, settlements, donations which benefit from the game of "overunderstanding". 